\title{
CTENOCEPHALIDES CHABAUDI sp. n. (SIPHONAPTERA, PULICIDAE), PUCE NOUVELLE DE LA FORÊT PRIMAIRE DU GABON
}

\author{
J. C. BEAUCOURNU* et 0 . BAIN**
}

RÉSUMÉ. Description de Ctenocephalides chabaudi sp. n. de la forêt primaire du Gabon. L'holotype (seul connu) est une femelle essentiellement caractérisée par sa ctenidie, son processus génal, sa chétotaxie et sa spermathèque.

L'hôte, sans doute accidentel, est Cephalophus dorsalis (Mamm., Bov.).

\section{Ctenocephalides chabaudi, sp. n. (Siphonaptera, Pulicidae),} a new flea from the primary forest in Gabon

SUMMARY. Ctenocephalides chabaudi sp. n. is described from the primary forest in Gabon. The holotype and only specimen is a female characterized by its ctenidia, its genal process, the pattern of setae on abdominal tergites and by its spermatheca. The host, presumably accidental, is Cephalophus dorsalis (Mammalia, Bovidae).

L'un de nous (O.B.) a récolté au Gabon, sur une antilope, un Ctenocephalides parfaitement insolite : bien qu'il ne soit représenté que par un exemplaire, et qui plus est, femelle, nous avons décidé de décrire cette puce devant l'originalité des caractères qu'elle présente 1 .

\section{Matériel de description}

Femelle hоLотуре sur Cephalophus dorsalis (Mamm., Bovidé, Céphalophiné), août 1981, Belinga (Gabon).

Cet exemplaire est déposé dans les collections du premier signataire.

* Laboratoire de Parasitologie (Entomologie médicale), Faculté de Médecine, F 35000 Rennes.

** Laboratoire des Vers, Muséum National d'Histoire Naturelle, Paris.

I. Cette récolte a été faite avec l'aide de l'Institut de Recherche en Écologie tropicale, à Makokou, et nous en remercions le Directeur, Paul Bosso.

Accepté le 2 novembre r98r. 
La station de récolte est en pleine forêt primaire équatoriale. Belinga est situé près de la frontière congolaise, au nord-est du Gabon, approximativement à $1^{\circ} 15^{\prime} \mathrm{N}$ $13^{\circ} 15^{\prime} \mathrm{E}$.

Nous sommes heureux de dédier ce taxon au Professeur Alain Chabaud (Muséum National d'Histoire Naturelle de Paris) en témoignage de respectueuse amitié.

\section{Description}

Capsule céphaligue : (fig. 1) front arrondi, Ctenidie génale de 7 dents, les deux antérieures subégales mais la première concave (et non convexe) dans sa moitié apicale. Tubercule préoral court. Eil grand. Une soie préorale courte; une soie préoculaire longue. Processus génal avec une épine apicale et une marge dorsale divergente, venant se raccorder à angle droit au niveau de l'apex. Trois rangées occipitales de soies, y compris la postérieure sub-marginale, composées respectivement de 1,1 et 5 soies.

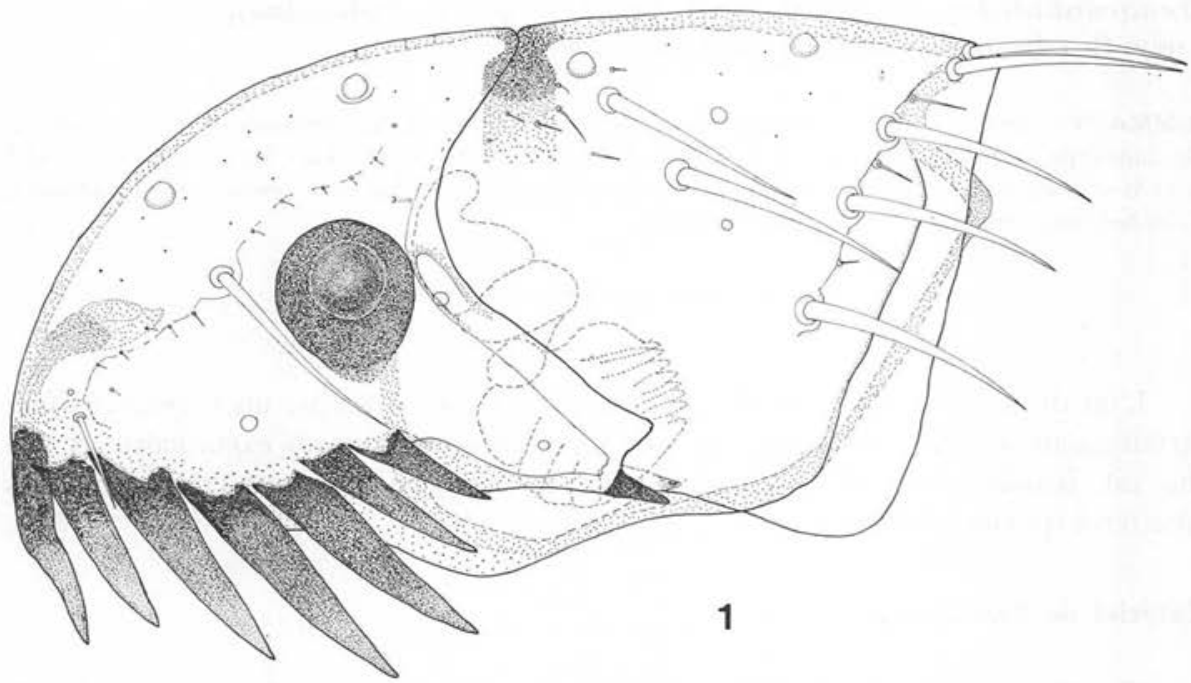

FIG. I. - Ctenocephalides chabaudi sp. n., holotype femelle, capsule céphalique.

Thorax : (fig. 2) Prothorax portant une rangée de 5 soies de chaque côté et une ctenidie de 14 dents au total; la dent dorsale est légèrement plus courte que le bord dorsal du segment ; la dent ventrale est au-dessus de la mi-hauteur du segment et de l'insertion de la pénultième soie prothoracique. Mesothorax et metathorax avec chacun une rangée de 5 soies (de chaque côté). Metepisternum portant 2 soies. Metepimeron : deux rangées de 7 soies chacune. Tibia III portant 6 encoches au bord dorsal. Setation et morphologie tarsale comme chez Ctenocephalides felis $s$. l., toute- 
fois la plus longue soie du $2^{\mathrm{e}}$ segment du tarse III atteint à peine l'apex du $4^{\mathrm{e}}$ segment. Griffes tarsales sans caractères adaptatifs particuliers comme il est de règle dans ce genre.

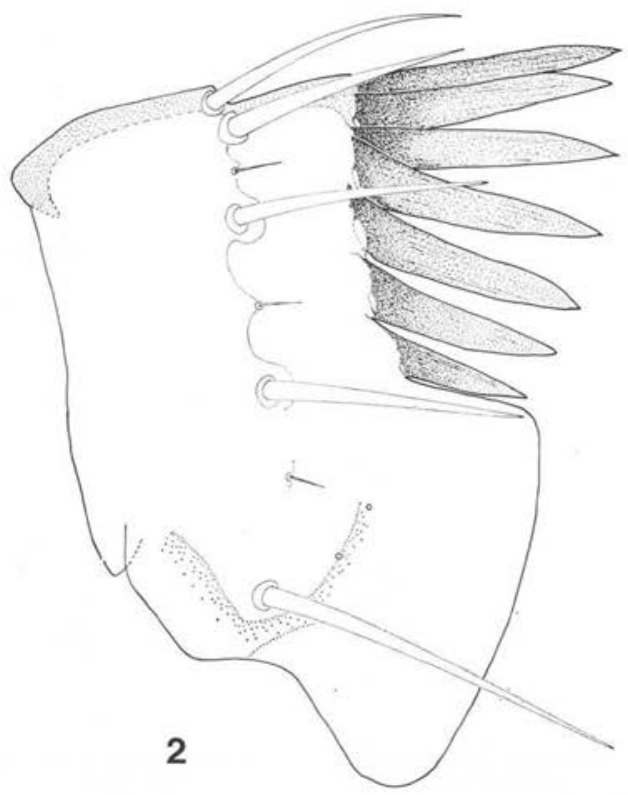

FIG. 2. - Ctenocephalides chabaudi sp. n., holotype femelle, prothorax.

Aвdomen : Stigmates subcirculaires, grands, intermédiaires en taille entre ceux de $C$. canis et ceux de $C$. felis $s$. $l$. Setation des tergites (pour chaque côté) comme suit de t. I à t. VII : 2 (+ 2 "rangs » de 1 soie situés plus antérieurement), $5,5,5,5,5$ et 4 . Tergite VIII aberrant ( fig. 3) : marge postérieure sinueuse et hyaline; 5 soies latérales et 4 marginales fortes, à la face externe ; 3 soies spiniformes sub-marginales et 2 marginales grêles, à la face interne ; stigmate très grand, légèrement supérieur au diamètre vertical de la plaque pygidiale. Sétation des sternites réduite : de st. II à st. VII on note, respectivement (pour chaque côté) $0,1,1,1,2$ et 2. Marge postérieure du sternite VII doucement concave avec un lobe ventral vestigial. Proctiger classique mais stylet anal long présentant une sétation originale : d'avant en arrière, 2 soies spiniformes, 2 très courtes et, apicalement, la grande soie classique (ici très courte, puisque de même longueur que le stylet) avec, à sa base, une soie minuscule et grêle.

SpermathÈque caractéristique : bulga grande, légèrement tronconique; hilla courte, faiblement arquée avec une papilla distincte ; ductus spermathecae débouchant dans l'angle postero-ventral de la bulga. 


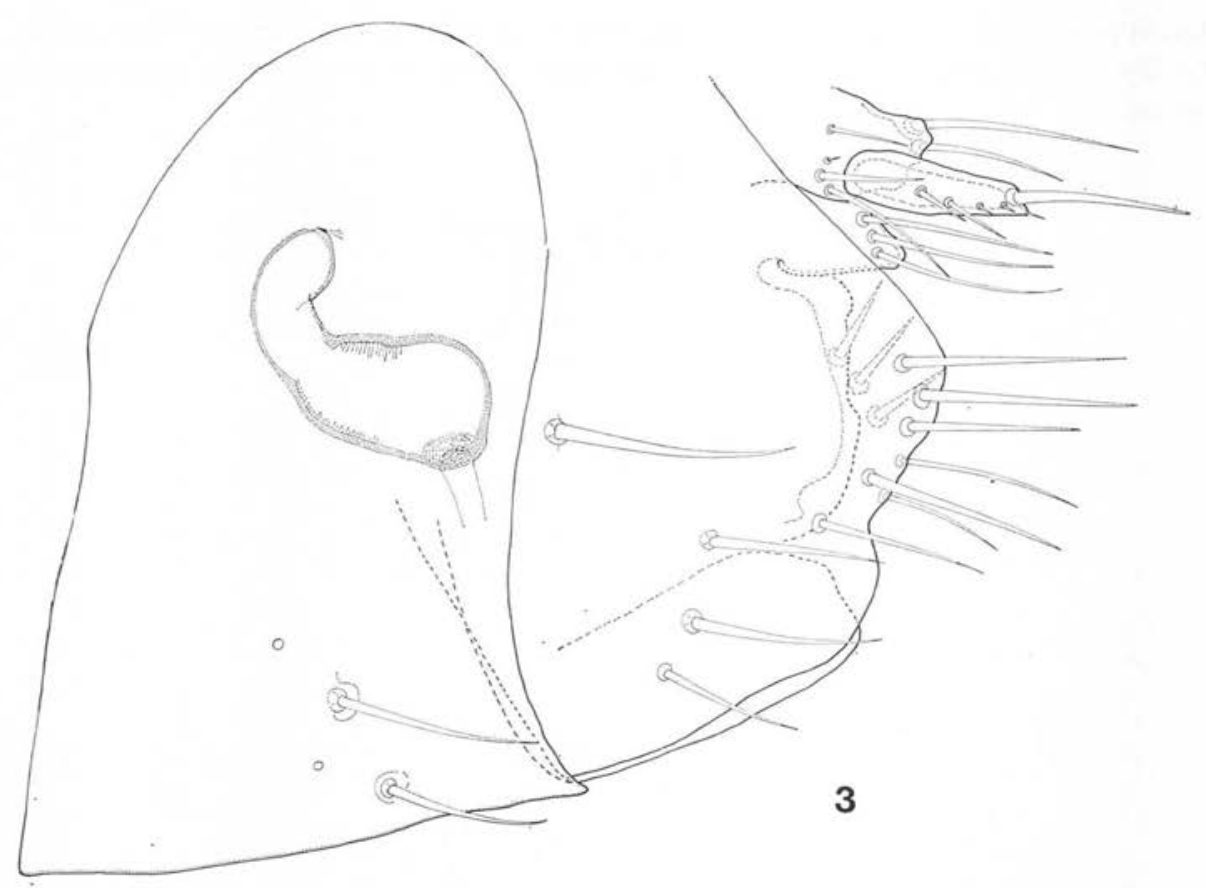

FIG. 3. - Ctenocephalides chabaudi sp. n., holotype femelle, sternite VII, tergite VIII, proctiger et spermathèque.

\section{Discussion}

Ctenocephalides chabaudi sp. n. se distinguera facilement de toutes les autres espèces connues par sa spermathèque mais aussi, bien que la variabilité intraspécifique ne puisse ici être appréciée, tout particulièrement par la morphologie du lobe génal, la sétation du tergite VIII, des sternites et du stylet anal.

Bien que l'absence de mâle rende impossible tout essai de filiation, on peut noter que la spermathèque, particulièrement originale, pourrait au maximum faire évoquer C. paradoxuri, décrit de Sri-Lanka (= Ceylan) où il parasite des Viverridés. Mise à part la forme et la sétation de la capsule céphalique, c'est d'ailleurs avec cette espèce que $C$. chabaudi présenterait, curieusement, le plus d'affinités (nombre de dents génales et pronotales, absence de soies sur le sternite II, sétation réduite du tergite VIII, brièveté de la plus longue soie du tarse II postérieur, insertion de l'avantdernière soie du prothorax par rapport à la dent inférieure de la ctenidie).

Bien que possible, l'attribution de $C$. chabaudi à un hôte bovidé, est sans doute accidentelle et, pour cette puce encore, c'est sans doute chez les Viverridés (Genetta spp.) qu'il faut rechercher, l'hôte normal. 